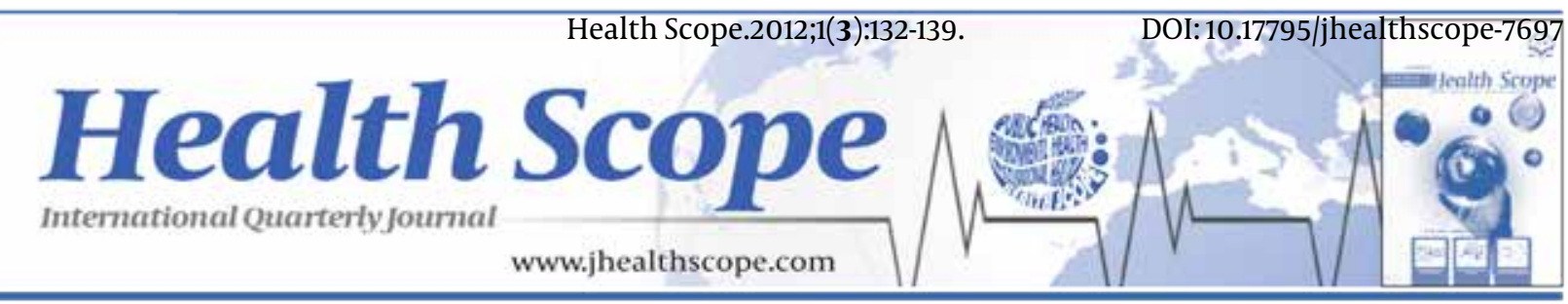

\title{
ManTRA for the Assessment of Musculoskeletal Risk Factors Associated With Manual Tasks in an Electric Factory
}

\author{
Seyyed Ali Moussavi-Najarkola ${ }^{{ }^{*}}$, Ramazan Mirzaei ${ }^{2}$ \\ ${ }^{1}$ Department of Occupational Hygiene, Collage of Health, Shahid Beheshti University of Medical Sciences, Tehran, IR Iran \\ 2 Department of Occupational Health, Health Promotion Research Center, Zahedan University of Medical Sciences, Zahedan, IR Iran
}

\section{A R T I C L E I N F O}

Article type:

Research Article

\section{Article history:}

Received: 11 Aug 2012

Revised: 24 Aug 2012

Accepted:30 Aug 2012

Keywords:

ManTRA

Risk Factors

Musculoskeletal System

\begin{abstract}
A B S T R A C T
Background: Work related musculoskeletal disorders (WMSDs) occur when occupational loads on anatomical structures are higher than the natural anatomical strength of the body structures. Therefore, assessment of the risk factors involved in manual tasks of electric factories can be useful to prevent WMSDs.

objectives: This study was conducted on Tehran Pars-electric factory to assess and prevent manual tasks risk factors causing WMSDs.

Patients and Methods: 673 workers, which 355 (52.7\%) were males (mean age: $38.9 \pm 7.3$ years) and $318(47.3 \%)$ were females (mean age: $25.6 \pm 9.8$ years) performing different manual tasks were recruited. Manual Tasks Risk Assessment (ManTRA) was set up to assess risks of anybody region disorder comprising wrist-hand, shoulder-arm, neck, back, and lower limb on subjects performing various manual tasks in Pars electric factory located in Tehran. Also Nordic Musculoskeletal Questionnaire (NMQ) was used to find prevalence of five mentioned body region disorders, to assay the accuracy of the results obtained by ManTRA.

Results: Total prevalence's of subjects with wrist-hand, shoulder-arm, neck back and lower limb disorders were obtained 556 (82.6\%), 352 (52.3\%), 238 (35.4\%), 454 (67.5\%), and $149(22.1 \%)$ respectively $(P=0.028)$. The highest cumulative risk was corresponded to wrist-hand, and after that were cumulative risks of back, shoulder-arm, neck, and lower limb respectively.

Conclusions: The results of NMQ and ManTRA were completely conforming. ManTRA is also a useful and an applicable tool to assess manual tasks risk in electric factories.
\end{abstract}

-Implication for health policy/practice/research/medical education:

This article was aimed to assess the risk factors of musculoskeletal disorders in an electric factory by "Manual Task Risk Assessment (ManTRA)". This ergonomic risk factor tool can be useful for the assessment of the manual tasks for ergonomists, occupational hygienists, occupational therapists, industrial designers, physiotherapists, rehabilitation specialists, and anthropologists.

- Please cite this paper as:

Moussavi-Najarkola SA, Mirzaei R. ManTRA for the Assessment of Musculoskeletal Risk Factors Associated With Manual Tasks in an Electric Factory. Health Scope. 2012; 1(3):132-9.

\footnotetext{
* Corresponding author: Seyyed Ali Moussavi Najarkola, Department of Occupational Hygiene, Collage of Health, Shahid Beheshti University of Medical Sciences(SBUMS), Daneshjou Boulevard, Daneshjou Square, Tabnak Street, Chamran Highway, Tehran, IR Iran. Tel: +98-2122432040-41, Fax: +98-2122432037, E-mail: mosavi58@gmail.com
} 


\section{Background}

Work-related musculoskeletal disorders (WMSDs) seem to be a main cause of disabilities and workers compensations (1). On the one hand, the estimated prevalence of WMSDs vary depending on assessment tools and exposed population characteristics (2). On the other hand, high occurrence of repetitive strain injuries in some tasks and jobs requires the need to identify the risk factors of these disorders at workplaces (3). Therefore, ergonomic risk factor assessment can help to prevent, minimize or eliminate WMSDs. Several ergonomic risk assessment tools have been developed for this intent (3). Most of the ergonomic quantitative risk assessment tools which have been proposed are only strictly applicable in very limited situations. Several attempts have been performed to assay the presented tools in situations to which they are uncovered (4). Also, the applicable problem of developing a tool to suit the ergonomic assessment of awkward risk factors presenting in manual tasks seems to be essential in ergonomic communications (4). Queensland Manual Tasks Advisory Standard defined the manual tasks simply as: "Manual tasks are the workplace activities requiring the use of force exerted by a person to grasp, manipulate, strike, throw, carry, move (lift, lower, push, pull), hold or restrain an object, load or body part" (5). Therefore, the necessity to an efficient and applicable ergonomic risk assessment tool in manual tasks seems to be evident (6). For simultaneously multiple risk factors presenting in WMSDs causation, a proposed or developed ergonomic risk assessment tool is essential to provide simultaneous assessment of multiple anatomical risk factors (4). An efficient risk assessment tool has to integrate the independent risk factors assessment of different body regions (4). The condition for an assessment tool to be appropriate to industries is to provide the guidelines respecting excessive threshold action levels (4). Thereby, a useful manual task risk assessment tool has to provide concurrent different risk factors assessment, and it must not need any special equipment, professional staff or advanced training (4).

Four categories of ergonomic posture-based risk assessment techniques have been developed to assess the physical exposure to WMSDs risk factors (7). These techniques are: observational methods (divided into penpaper based observational and video-taping or computer-aided observational methods including OWAS, RULA, REBA, HAMA, PLIBLE, OCRA, Posture targeting, Posture gram, Gil \& Tunes method, QEC, SI, PEO, ARBAN, VIRA, ROTA, TRAC, HARBO, PEO, Radwin-Yen, Wells, LUBA, and other methods), instrumental or direct techniques, self-report techniques and psycho-physiological techniques $(7,8)$. All aforementioned techniques have failed to meet the criteria of an appropriate manual tasks risk assessment tool (9). Manual Tasks Risk Assessment
(ManTRA) as a manual tasks risk assessment tool seems to be an appropriate technique to assess ergonomic risk factors associated with manual tasks of electric factories.

\section{Objectives}

The study was conducted to investigate whether ManTRA is useful and applicable, not only for evaluating ergonomic risk factors, but also for determining cumulative risks of body regions by providing ManTRA scoring system in such manual tasks.

\section{Patients and Methods}

673 workers, which 355 (52.7\%) were males (mean age: $38.9 \pm 7.3$ years) and 318 (47.3\%) were female, performing different manual tasks in television $\&$ radio production lines of Pars electric factory located in Tehran were recruited. A new presented risk assessment tool called the Manual Tasks Risk Assessment (ManTRA) was used to gain the exposure assessment of musculoskeletal risk factors presented in manual tasks $(4,6)$. Nordic Musculoskeletal. Questionnaire (NMQ) was also completed by all the workers examined to find the prevalence of musculoskeletal disorders of anybody regions surveyed by ManTRA tool (10).

\subsection{Manual Tasks Risk Assessment (ManTRA)}

Manual Tasks Risk Assessment (ManTRA) as an ergonomic risk assessment tool was proposed as a common proposal among University of Queensland, Curtin University of Technology, and Queensland Division of Workplace Health and Safety in 2003 (9). This technique was planned to assess the WMSDs risk level present in different workplace manual tasks $(4,9)$. ManTRA was conceptually based on Strain Index (SI) and Quick Exposure Check (QEC) $(4,9)$. ManTRA is required to total exposure assessment of five body regions to: repetition; exertion; awkward postures and vibration $(4,9)$. Possible proposed action level thresholds are then presented for exertion only; combination of exertion and awkwardness; and total exposure only. Main advantage of ManTRA can be attributed to providing the simultaneous interactions among different risk factors for anybody region $(4,9)$. ManTRA provides criteria for manual tasks risk assessment without the need to prior training and can be useful for workplace health and safety inspectors (9). The most important purpose of ManTRA is to provide an exposure risk assessment to musculoskeletal risk factors of manual tasks in workplaces and industries (4, 9). Similar to other ergonomic tools, a team work risk assessment comprising the employees performing the manual tasks and the assessors assessing the manual task can be helpful to achieve a correct assessment (4, 9) Action level thresholds imply on the assessors 'judg- 
ments regarding the need for implementation of control measures (9). Combination of the force and speed leads to the exertion risk, and combination of the cycle time and duration gives the repetition risk, and finally the cumulative risk score for anybody region can be gained by summing total time, repetition, exertion, awkwardness and vibration which can be ranged from 5 to 25 (9). Prioritization of manual tasks-related control measures should be necessary as for anybody region, the combined exertion risk is 5; or the combined exertion and awkwardness risk is 8 or greater; or the combined cumulative risk is 15 or greater (9).

\subsection{Nordic Musculoskeletal Questionnaire (NMQ)}

There are several standardized musculoskeletal questionnaires which can be useful to survey symptoms and pains of WMSDs. One of earliest standardized musculoskeletal questionnaires is 'Nordic Musculoskeletal Questionnaire (NMQ)'. This questionnaire has been proposed and developed by Kuorinka and his colleagues from Occupational Health Institute in Nordic (Scandinavian) Countries in 1987 (10,11). This questionnaire can well suit the surveillance intents, but complementary development of an appropriate standardized ergonomic risk assessment tool to ascertain symptombased musculoskeletal syndromes (11). This questionnaire can also be served as a useful tool for gathering data and information related to musculoskeletal disorders like prevalence, incidence and epidemiology of disorders $(10,11)$.

\section{Results}

673 subjects aged $19-49$ years ( $32.3 \pm 11.9$ years) performing different manual tasks in Pars electric factory were studied, including 355 (52.7\%) males aged 25-49 years (38.9 \pm 7.3 years), and 318 (47.3\%) females aged $19-33$ years (25.6 \pm 9.8 years). The male/female ratio was 1:1.1 (Table 1). The list of the tasks assessed by completing NMQ and implementing ManTRA has been shown in Table 2 . Most of workers were involved in task of chassis control aided monitor with the total frequency of 31 subjects (15 males and 16 females), and the least was related to the task of tape assembling with total frequency of 14 subjects ( 8 males and 6 females). Also the table showed that the highest and the lowest mean age in male popu- lations was related to the tasks of bobbin wrapping and placing TV image lamp in cabin correspondingly. On the other hand, the highest and the lowest mean age in female populations were related to the tasks of radio assembling and TV channel regulating respectively. The occurrence of 'anamnestic cases' by task type and body regions disorders has been demonstrated in Table 3 was wrist-hand, back, shoulder-arm, neck and lower limb. The prevalence of the wrist-hand disorders had the highest rates among other body regions and after that were the back, shoulder-arm, neck and lower limb disorders respectively. Thereby, it is seen from the table that the total prevalence of wrist-hand, shoulder-arm, neck, back and lower limb disorders were obtained 556 (82.6\%), 352 (52.3\%), 238 (35.4\%), 454 (67.5\%), and 149 (22.1\%) respectively $(P=0.028)$. According to Table 3 , the most and the least prevalence for most body regions disorders were related to the tasks of "TV frame preparing" and "tape assembling" respectively.

The task data and information for the assessment of risk factors o calculate cumulative risks (setting up the Manual asks Risk Assessment tool) has been illustrated in Table 4. As observed, five mentioned risk factors (include: total time (Tr), repetition risk (Rr), exertion risk (Er), awkwardness (A), and vibration (V)) were surveyed and assessed for five aforesaid body regions. The corresponding data is achievable from the table. Only submitted final scores of any risk factors for avoiding complicacy and better understanding. The table also shows that the highest scores were related to the wristhand region and after that were back, shoulder-arm, neck, and lower limb regions. The combined cumulative risk calculated for anybody region assessed in various tasks and comparison of the calculated cumulative risk to the predicted threshold limit value (TLV) (15 or greater) for proposing further actions for body regions that their calculated cumulative risks exceeded TLV, has been shown in Table 5. As seen, the highest cumulative risks calculated in various tasks were related to the wrist-hand region and after it, back, shoulderarm, neck, and lower limb regions had been gained the highest cumulative risks orderly. There was only a task that cumulative risks calculated for any five body regions exceeded TLV and there must be placed in the prioritization of tasks for control and performing further actions and measurements. The correspond-

\begin{tabular}{lcccc}
\hline \multicolumn{2}{l}{ Table 1. Distribution of Studied Workers for Gender and Age } \\
\hline \multicolumn{1}{l}{ Workers, No. (\%) } & \multicolumn{3}{c}{ Age } \\
\cline { 2 - 5 } & & Minimum & Maximum & Mean \pm SD \\
\hline Male & $355(52.7)$ & 25 & 49 & $38.9 \pm 7.3$ \\
Female & $318(47.3)$ & 19 & 33 & $25.6 \pm 9.8$ \\
Total & $673(100)$ & 19 & 49 & $32.3 \pm 11.9$ \\
\hline
\end{tabular}




\begin{tabular}{|c|c|c|c|}
\hline & Task Code & Worker Frequency, No. (\%) & Worker Age, Mean \pm SD \\
\hline Radio chassis assembling & $\mathrm{T}_{1}$ & $21(3.12)$ & $38.3 \pm 1.22$ \\
\hline TV chassis assembling & $\mathrm{T}_{2}$ & $22(3.26)$ & $39.6 \pm 1.13$ \\
\hline Electrical parts soldering & $\mathrm{T}_{3}$ & $19(2.82)$ & $31.8 \pm 3.89$ \\
\hline Observational chassis control & $\mathrm{T}_{4}$ & $25(3.71)$ & $36.2 \pm 1.76$ \\
\hline Chassis control aided ACT computer & $\mathrm{T}_{5}$ & $19(2.82)$ & $30.8 \pm 2.33$ \\
\hline Chassis control aided monitor & $\mathrm{T}_{6}$ & $31(4.61)$ & $35.9 \pm 4.91$ \\
\hline Tuner production & $\mathrm{T}_{7}$ & $22(3.26)$ & $40.1 \pm 3.7$ \\
\hline Bobbin wrapping & $\mathrm{T}_{8}$ & $21(3.12)$ & $30.3 \pm 4.61$ \\
\hline Loud speaker production & $\mathrm{T}_{9}$ & $20(2.97)$ & $37.7 \pm 3.57$ \\
\hline Coil Gaznik production & $\mathrm{T}_{10}$ & $19(2.82)$ & $40.3 \pm 2.11$ \\
\hline Electric parts repairing & $\mathrm{T}_{11}$ & $25(3.71)$ & $37.1 \pm 2.65$ \\
\hline TV frame preparing & $\mathrm{T}_{12}$ & $28(4.16)$ & $41.6 \pm 3.13$ \\
\hline TV image lamp preparing & $\mathrm{T}_{13}$ & $27(4.01)$ & $33.7 \pm 2.71$ \\
\hline Placing TV image lamp in cabin & $\mathrm{T}_{14}$ & $21(3.12)$ & $45.1 \pm 2.12$ \\
\hline Placing chassis in cabin & $\mathrm{T}_{15}$ & $21(3.12)$ & $36.2 \pm 3.10$ \\
\hline TV technical control & $\mathrm{T}_{16}$ & $19(2.82)$ & $43.2 \pm 3.8$ \\
\hline Radio technical control & $T_{17}$ & $23(3.42)$ & $41.1 \pm 2.01$ \\
\hline Placing radio back cover & $\mathrm{T}_{18}$ & $17(2.7)$ & $39.6 \pm 1.08$ \\
\hline Placing TV back cover & $\mathrm{T}_{19}$ & $19(2.82)$ & $40.8 \pm 2.22$ \\
\hline Manual controlling and tapping & $\mathrm{T}_{20}$ & $17(2.7)$ & $41.2 \pm 2.16$ \\
\hline TV cabin preparing & $\mathrm{T}_{21}$ & $21(3.12)$ & $40.6 \pm 1.67$ \\
\hline Radio and tape record production & $\mathrm{T}_{22}$ & $21(3.12)$ & $35.4 \pm 1.88$ \\
\hline Resistance inserting & $\mathrm{T}_{23}$ & $18(2.67)$ & $38.9 \pm 2.72$ \\
\hline Wires connecting & $\mathrm{T}_{24}$ & $21(3.12)$ & $35.3 \pm 3.29$ \\
\hline $\mathrm{IC}_{\mathrm{s}}$ inserting & $\mathrm{T}_{25}$ & $17(2.7)$ & $32.7 \pm 2.19$ \\
\hline TV assembling & $\mathrm{T}_{26}$ & $17(2.7)$ & $30.5 \pm 3.44$ \\
\hline Radio assembling & $\mathrm{T}_{27}$ & $21(3.12)$ & $38.8 \pm 2.84$ \\
\hline Radio waves regulating & $\mathrm{T}_{28}$ & $21(3.12)$ & $38.3 \pm 3.14$ \\
\hline TV channel regulating & $\mathrm{T}_{29}$ & $26(3.86)$ & $33.4 \pm 3.17$ \\
\hline Tape assembling & $\mathrm{T}_{30}$ & $14(2.08)$ & $39.7 \pm 2.12$ \\
\hline Radio final control & $\mathrm{T}_{31}$ & $20(2.97)$ & $39.1 \pm 1.07$ \\
\hline TV final control & $\mathrm{T}_{32}$ & $20(2.97)$ & $43.1 \pm 1.45$ \\
\hline
\end{tabular}

ing task was radio technical control. The tasks such as radio chassis assembling, chassis control aided monitor, tuner production, and bobbin wrapping had only cumulative risks exceeded TLV for wrist-hand that the tasks must be placed in the end of prioritization classification for correcting work conditions. The highest cumulative risks for wrist-hand (24), shoulder-arm (19), neck (16), back (19), and lower limb (17) were related to the tasks of loud speaker production, radio assembling, TV channel regulating, loudspeaker production, and radio technical control respectively. Also the lowest cumulative risks for wrist-hand (15), shoulderarm (11), neck (7), back (11), and lower limb (7) were corresponded to the tasks of TV technical control, bobbin wrapping, , chassis control aided monitor, and chassis control aided ACT computer respectively.

\section{Discussion}

NMQ results showed the highest prevalence about wristhand, back, shoulder-arm, neck and lower limb respectively. Therefore, very high prevalence of the disorders for anybody regions of workers performing different manual 


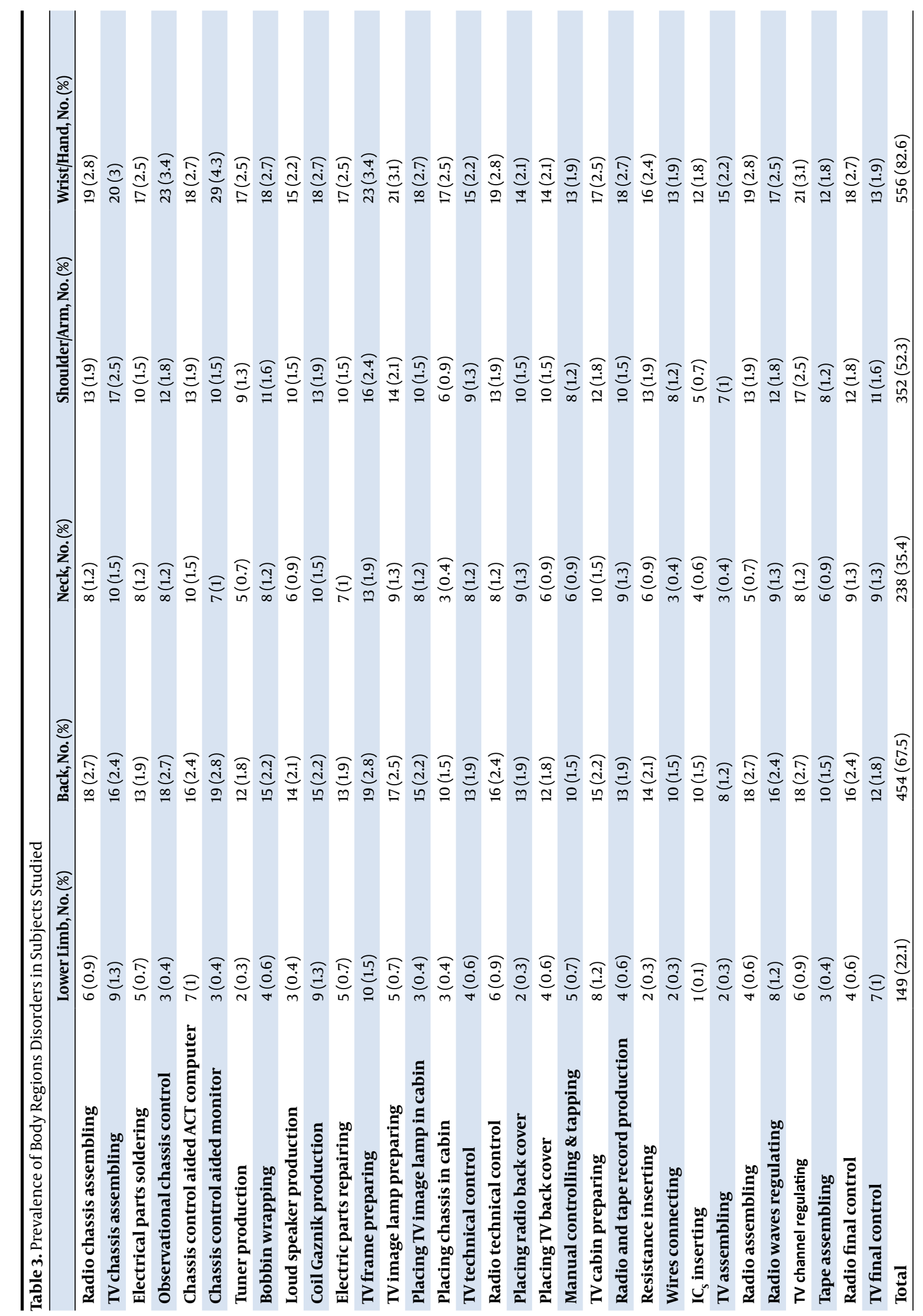




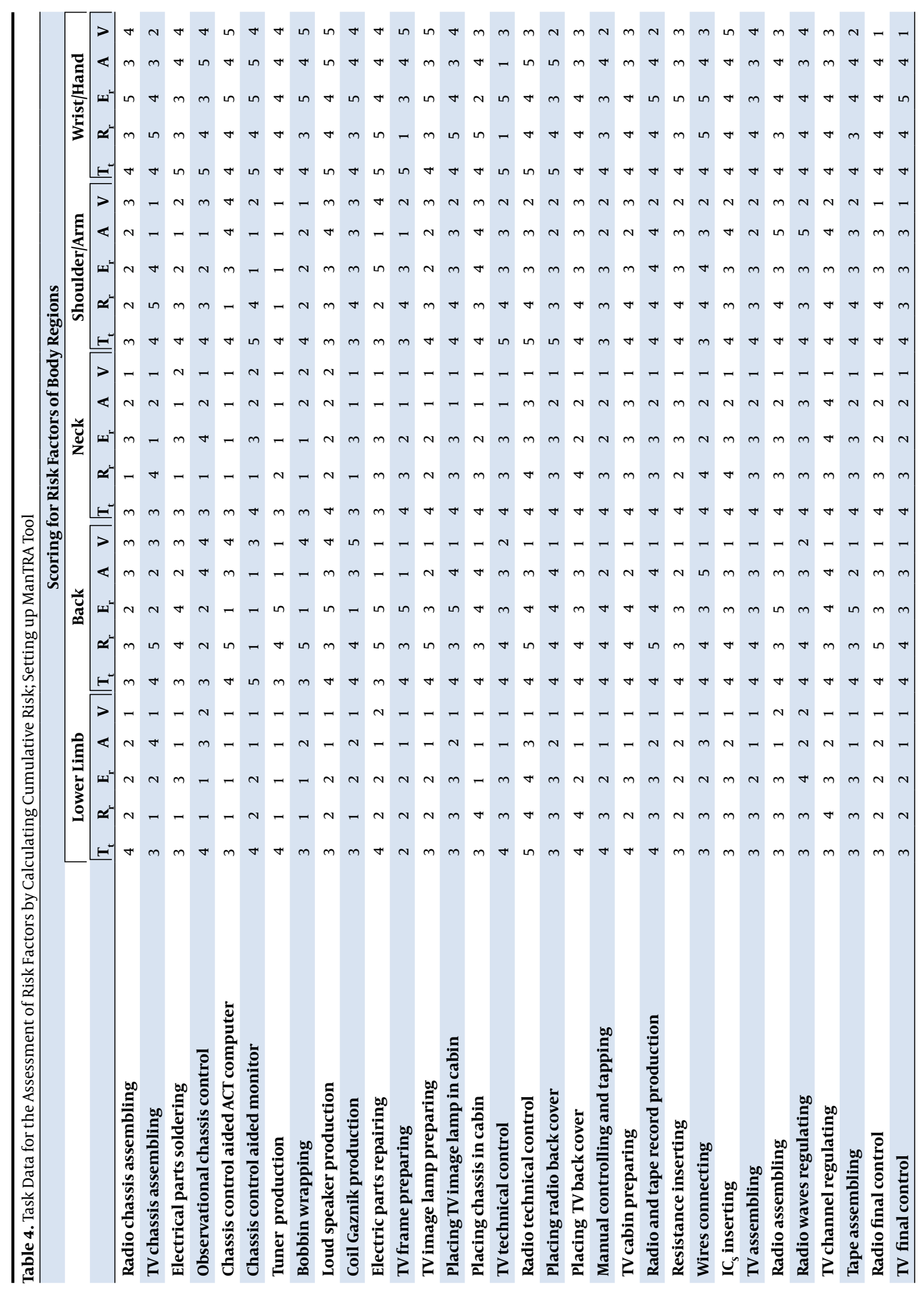




\begin{tabular}{|c|c|c|c|c|c|}
\hline \multirow[t]{2}{*}{ Task Type } & \multicolumn{5}{|c|}{ Cumulative Risk } \\
\hline & Lower Limb & Back & Neck & Shoulder/Arm & Wrist/Hand \\
\hline Radio chassis assembling & 11 & 14 & 10 & 12 & 19 \\
\hline TV chassis assembling & 11 & 16 & 11 & 15 & 18 \\
\hline Electrical parts soldering & 9 & 16 & 10 & 12 & 19 \\
\hline Observational chassis control & 11 & 15 & 11 & 13 & 21 \\
\hline Chassis control aided ACT computer & 7 & 17 & 7 & 16 & 22 \\
\hline Chassis control aided monitor & 10 & 11 & 12 & 13 & 23 \\
\hline Tuner production & 8 & 14 & 8 & 8 & 20 \\
\hline Bobbin wrapping & 8 & 14 & 9 & 11 & 21 \\
\hline Loud speaker production & 9 & 19 & 12 & 16 & 23 \\
\hline Coil Gaznik production & 9 & 17 & 9 & 16 & 20 \\
\hline Electric parts repairing & 11 & 15 & 11 & 15 & 22 \\
\hline TV frame preparing & 8 & 14 & 11 & 13 & 18 \\
\hline TV image lamp preparing & 9 & 15 & 10 & 14 & 20 \\
\hline Placing TV image lamp in cabin & 12 & 17 & 12 & 16 & 20 \\
\hline Placing chassis in cabin & 10 & 16 & 11 & 18 & 18 \\
\hline TV technical control & 12 & 16 & 12 & 17 & 15 \\
\hline Radio technical control & 17 & 17 & 15 & 17 & 21 \\
\hline Placing radio backcover & 12 & 17 & 13 & 15 & 19 \\
\hline Placing TV backcover & 12 & 15 & 13 & 17 & 18 \\
\hline Manual controlling \& tapping & 11 & 15 & 12 & 13 & 16 \\
\hline TV cabin preparing & 11 & 15 & 14 & 16 & 18 \\
\hline Radio and tape record production & 13 & 18 & 13 & 18 & 19 \\
\hline Resistance inserting & 10 & 13 & 13 & 16 & 18 \\
\hline Wires connecting & 12 & 17 & 13 & 16 & 21 \\
\hline $\mathrm{IC}_{\mathrm{s}}$ inserting & 12 & 15 & 14 & 16 & 21 \\
\hline TV assembling & 10 & 15 & 13 & 14 & 18 \\
\hline Radio assembling & 12 & 16 & 13 & 19 & 18 \\
\hline Radio waves regulating & 14 & 16 & 14 & 17 & 19 \\
\hline TV channel regulating & 13 & 16 & 16 & 17 & 18 \\
\hline Tape assembling & 11 & 15 & 13 & 16 & 17 \\
\hline Radio final control & 10 & 16 & 12 & 15 & 17 \\
\hline TV final control & 9 & 15 & 12 & 14 & 18 \\
\hline
\end{tabular}

task were evident. ManTRA showed notably the similar results to NMQ. The highest cumulative risks for wrist-hand, shoulder-arm, neck, back and lower limb were allocated to the loud speaker production, radio assembling, TV channel regulating, loudspeaker production, and radio technical control respectively. Therefore, further action measures and controls should be considered to these tasks to optimize risk factors involved in WMSDs in manual tasks. While the lowest cumulative risks for five body regions of wrist-hand, shoulder-arm, neck, back, and lower limb were also related to the tasks of TV technical control, bobbin wrapping, chassis control aided ACT computer, chassis control aided monitor, and chassis control aided ACT computer respectively. Radio technical control was a unique task which its cumulative risks calculated for all five body regions were excessive TLVs, so that ergonomic control measures must be implemented primarily for this task before any further measurements.

ManTRA seems to be an appropriate tool for the evaluation of ergonomic risk factors leading to WMSDs in manual tasks, since the gained results from this tool are consistent with biomechanical evidence which support the theory that prolonged and repetitive exposure to ergonomic risk factors can lead to WMSDs in manual tasks (9). These awkward risk factors in ManTRA are mainly consisted of forceful exertions, awkward and static postures and vibration which can help to accumulate the risks of WMSDs (9). Determination of body structural loading excessive TLVs is difficult due to the body anatomical and biomechanical complexities. Therefore, ManTRA is an appropriate assessment 
tool for combining separate interactions among different involved risk factors to an integrated cumulative risk which have direct association with epidemiological data of WMSDs prevalences $(4,9)$. Unique advantage of ManTRA is its capability to assess the interactions which may be occurred among physical risk factors and also among physical, environmental and psychophysical risk factors in manual tasks (9). However, defining exact TLVs for ergonomic risk factors involved in manual tasks is not only very difficult, but also somewhat impossible $(4,9)$. Therefore, strict precautions should be considered in interpreting the cumulative risk scores obtained from ManTRA (9). Appropriate training to ManTRA users as well as employees' and management' contribution to task analysis and risk assessment process can help to gain the precise risk assessment results $(4,9)$. ManTRA suggests that the tasks with cumulative risk scores excessive TLVs should be considered to suffer further measurements or actions to reduce obtained cumulative risk scores to TLVs to prevent WMSDs occurrences $(9,12)$. Preventive measures should be exerted on the manual tasks that have cumulative risk scores excessive TLVs for minimizing WMSDs occurrences of anybody regions (13). Preventive measures on manual tasks excessive TLVs can be categorized into three classes including structural, organizational and educational measures (13). These assorted measures should be implemented whenever any manual task risk assessment assessed by ManTRA showed the cumulative risk scores excessive TLVs (13). Structural measures generally include redesigning the manual task area and ergonomically furnishing the layout of task components, hand tools and equipments (13). Structural measures can lead to modify the risk factors of excessive force exertion and poor postures (13). Organizational measures are mainly referred to task redesigning (including tasks distribution, task speeds and task rests or breaks) (13). Organizational measures can result to correct the risk factors related to frequent activities or repetition, insufficient recovery times and extremely lengthy tasks (13). Organizational measures can be possible by lowering the the frequency of repetitive tasks to TLVs, identifying a suitable ergonomic plan and risk reduction schedule and monitoring the cost-effectiveness of implemented plans (13). Educational measures use the special education and training contents for fitting the tasks demands to the employees' needs (13).

\section{Acknowledgments}

None declared.

\section{Authors' Contribution}

Authors work equally.

\section{Financial Disclosure}

None declared.

\section{Funding/Support}

None declared.

\section{References}

1. Buckle PW, Devereux JJ. The nature of work-related neck and upper limb musculoskeletal disorders. Appl Ergon. 2002;33(3):207-17.

2. Moussavi Najarkola SA. Concise Exposure and Damage Indicators for Predicting Foreseeable Effects Of Work-Related Upper Limb Disorders. Iranian J Publ Health. 2006;35(4):75-8.

3. Ketola R, Toivonen R, Viikari-Juntura E. Interobserver repeatability and validity of an observation method to assess physical loads imposed on the upper extremities. Ergonomics. 2001;44(2):119-31.

4. Division of Workplace Health and Safety D. Manual tasks advisory standard. Department of Industrial Relations. Queensland.pp. 3-7.; 1999; Available from: http://www.deir.qld. gov.au/workplace/index.htm.

5. Genaidy AM, al-Shedi AA, Shell RL. Ergonomic risk assessment: preliminary guidelines for analysis of repetition, force and posture. J Hum Ergol (Tokyo). 1993;22(1):45-55.

6. Swift MB, Cole DC, Beaton DE, Manno M. Health care utilization and workplace interventions for neck and upper limb problems among newspaper workers. J Occup Environ Med. 2001;43(3):265-75.

7. Li G, Buckle P. Current techniques for assessing physical exposure to work-related musculoskeletal risks, with emphasis on posture-based methods. Ergonomics. 1999;42(5):674-95.

8. Moussavi-Najarkola SA. Assessment of risk factors of upper extremity musculoskeletal disorders (UEMSDS) by OCRA method in repetitive tasks. Iranian J Publ Health. 2006;35(1):68-74.

9. Burgess-Limeric R. Issues associated with force and weight limits and associated theshold limit values in the physical handling work environment.Unpublished document. National Occupational Health \& Safety Commission, Queensland. pp. 1-6. 2003; Available from: http://ergonomics.uq.edu.au/download/threshold.pdf.

10. Kuorinka I, Jonsson B, Kilbom A, Vinterberg H, Biering-Sorensen F, Andersson G, et al. Standardised Nordic questionnaires for the analysis of musculoskeletal symptoms. Appl Ergon. 1987;18(3):233-7.

11. Garg A, Moore JS. Prevention strategies and the low back in industry. Occup Med. 1992;7(4):629-40.

12. Battevi N, Menoni O, Vimercati C. The occurrence of musculoskeletal alterations in worker populations not exposed to repetitive tasks of the upper limbs. Ergonomics. 1998;41(9):1340-6.

13. Bergamasco R, Girola C, Colombini D. Guidelines for designing jobs featuring repetitive tasks. Ergonomics. 1998;41(9):1364-83. 